\title{
BIBECHANA
}

A Multidisciplinary Journal of Science, Technology and Mathematics

ISSN 2091-0762 (online)

Journal homepage: http://nepjol.info/index.php/BIBECHANA

\section{On The Eneström-Kakeya Theorem}

A. Liman ${ }^{1}$, Tawheeda Rasool ${ }^{1}$, W.M Shah ${ }^{2}$

${ }^{1}$ Department of Mathematics, National Institute of Technology, India -190006

${ }^{2}$ Department of Mathematics, Jammu and Kashmir Institute of Mathematical Sciences-190008

e-mail:wmshah@rediffmail.com, abliman22@yahoo.com

Article history: Received 25 September, 2013; Accepted 23 November, 2013

\section{Abstract}

In this paper we present some interesting generalizations of Eneström-Kakeya type results concerning the location of zeros of a polynomial in the complex plane. We relax the hypothesis and put less restrictive conditions on the coefficients of the polynomial, and thereby generalize some classical results.

Keywords: polynomials, zeros, Eneström-Kakeya theorem

\section{Introduction}

The following result known as Eneström-Kakeya Theorem (for Reference see [1-3]) is well-known in the theory of distribution of zeros of polynomials.

Theorem A. If $P(z)=\sum_{j=0}^{n} a_{j} z^{j}$ is a polynomial of degree $\mathrm{n}$ such that

$$
a_{n} \geq a_{n-1} \geq \ldots \geq a_{1} \geq a_{0}>0,
$$

then all the zeros of $P(\mathrm{z})$ lie in $|z| \leq 1$.

Joyal, Labelle and Rahman [4] dropped the restriction on the hypothesis that all the coefficients be positive and proved the following:

Theorem B. If $P(z)=\sum_{j=0}^{n} a_{j} z^{j}$ is a polynomial of degree $\mathrm{n}$ such that

$$
a_{n} \geq a_{n-1} \geq \ldots . . \geq a_{1} \geq a_{0}
$$

then all the zeros of $P(\mathrm{z})$ lie in

$$
|z| \leq \frac{a_{n}-a_{0}+\left|a_{0}\right|}{\left|a_{n}\right|} .
$$

More recently Aziz and Zargar [5] relaxed the hypothesis of Eneström Kakeya theorem and proved some interesting extensions of Theorem B. In fact they proved

THEOREM C. If $P(z)=a_{n} z^{n}+a_{n-1} z^{n-1}+\ldots+a_{1} z+a_{0}$, is a polynomial of degree $\mathrm{n}$ such that for some $k \geq 1$, 


$$
k a_{n} \geq a_{n-1} \geq \ldots \geq a_{2} \geq a_{1} \geq a_{0},
$$

then all the zeros of $\mathrm{P}(\mathrm{z})$ lie in

$$
|z+k-1| \leq \frac{k a_{n}-a_{0}+\left|a_{0}\right|}{\left|a_{n}\right|} .
$$

More recently, Shah and Liman [6] while assuming the coefficients of the polynomials to be complex numbers among other things proved the following generalization of Theorem C.

Theorem D. Let $P(z)=\sum_{j=0}^{n} a_{j} z^{j}$ be a polynomial with complex coefficients. If $\operatorname{Re} a_{j}=\alpha_{j}$ and $\operatorname{Im} a_{j}=\beta_{j}$ for $j=0,1,2, \ldots, n, a_{n} \neq 0$ such that for some $k \geq 1$

$$
\begin{aligned}
& k \alpha_{n} \geq \alpha_{n-1} \geq \alpha_{n-2} \geq \ldots \geq \alpha_{1} \geq \alpha_{0}, \\
& \beta_{n} \geq \beta_{n-1} \geq \ldots \geq \beta_{1} \geq \beta_{0}>0,
\end{aligned}
$$

then all the zeros of $P(z)$ lie in

$$
\left|z+\frac{\alpha_{n}}{a_{n}}(k-1)\right| \leq \frac{k \alpha_{n}-\alpha_{0}+\left|\alpha_{0}\right|+\beta_{n}}{\left|a_{n}\right|}
$$

In this paper, we prove some Enström-Kakeya type results which besides generalizing some earlier results proved in this direction, include Theorem B, Theorem C and Theorem D as special cases. We start by proving the following:

\section{Theorems and Proofs}

Theorem 1: Let $P(z)=\sum_{j=0}^{n} a_{j} z^{j}$ be a polynomial of degree n. If $\operatorname{Re} a_{j}=\alpha_{j}, \operatorname{Im} a_{j}=\beta_{j}$ for $j=0,1,2, \ldots, n, a_{n} \neq 0$ and if for some positive integer $\lambda \leq n$ and $k \geq 1$

$$
k^{n-\lambda+1} \alpha_{n} \geq k^{n-\lambda} \alpha_{n-1} \geq k^{n-\lambda-1} \alpha_{n-2} \geq \ldots \geq k \alpha_{\lambda} \geq \alpha_{\lambda-1} \geq \ldots \geq \alpha_{1} \geq \alpha_{0}
$$

then all the zeros of $\mathrm{P}(\mathrm{z})$ lie in

$$
|z+k-1| \leq \frac{\left\{\alpha_{n}-\alpha_{0}+\left|\alpha_{0}\right|+(k-1)\left(\sum_{j=\lambda}^{n}\left(\alpha_{j}+\left|\alpha_{j}\right|\right)-\left|\alpha_{n}\right|\right)+2 \sum_{j=0}^{n}\left|\beta_{j}\right|\right\}}{\left|\alpha_{n}\right|} .
$$

If we take $\lambda=n$ we obtain the following :

Corollary 1.1: Let $P(z)=\sum_{j=0}^{n} a_{j} z^{j}$ be a polynomial of degree $\mathrm{n}$ with $\operatorname{Re} a_{j}=\alpha_{j}, \operatorname{Im} a_{j}=\beta_{j}$ for $j=0,1,2, \ldots, n, a_{n} \neq 0$ and if for some $k \geq 1$

$$
k \alpha_{n} \geq \alpha_{n-1} \geq \alpha_{n-2} \geq \ldots \geq \alpha_{1} \geq \alpha_{0}
$$

then all the zeros of $\mathrm{P}(\mathrm{z})$ lie in 


$$
|z+k-1| \leq \frac{k \alpha_{n}-\alpha_{0}+\left|\alpha_{0}\right|+2 \sum_{j=0}^{n}\left|\beta_{j}\right|}{\left|\alpha_{n}\right|} .
$$

For $\lambda=n-1$ and assuming all coefficients real and positive in Theorem 1, we obtain the following Corollary 1.2: Let $P(z)=\sum_{j=0}^{n} a_{j} z^{j}$ be a polynomial of degree $\mathrm{n}$ and if for some $k \geq 1$

$$
k^{2} a_{n} \geq k a_{n-1} \geq a_{n-2} \geq \ldots \geq a_{1} \geq a_{0}>0
$$

then all the zeros of $\mathrm{P}(\mathrm{z})$ lie in

$$
|z+k-1| \leq k+2(k-1) \frac{a_{n-1}}{a_{n}}
$$

or equivalently

$$
|z+k-1| \leq k(2 k-1)
$$

Remark 1.1: Theorem $\mathrm{C}$ is a special case of Theorem 1, if we take all coefficients real and $\lambda=n$. Further for all coefficients real, $\lambda=n$ and $k=1$ we obtain Theorem B.

Theorem 2: Let $P(z)=\sum_{j=0}^{n} a_{j} z^{j}$ be a polynomial of degree n. If $\operatorname{Re} a_{j}=\alpha_{j}$ and $\operatorname{Im} a_{j}=\beta_{j}$ for $j=0,1,2, \ldots, n, a_{n} \neq 0$ and if for some positive integer $\lambda \leq n$ and $k \geq 1$

$$
k^{n-\lambda+1} \beta_{n} \geq k^{n-\lambda} \beta_{n-1} \geq k^{n-\lambda-1} \beta_{n-2} \geq \ldots \geq k \beta_{\lambda} \geq \beta_{\lambda-1} \geq \ldots \geq \beta_{1} \geq \beta_{0}
$$

then all the zeros of $\mathrm{P}(\mathrm{z})$ lie in

$$
|z+k-1| \leq \frac{\left\{\beta_{n}-\beta_{0}+\left|\beta_{0}\right|+(k-1)\left(\sum_{i=\lambda}^{n}\left(\beta_{j}+\left|\beta_{j}\right|\right)-\left|\beta_{n}\right|\right)+2 \sum_{j=0}^{n}\left|\alpha_{j}\right|\right\}}{\left|\beta_{n}\right|}
$$

Next as a generalization of Therorem $\mathrm{D}$, we prove

Theorem 3: Let $P(z)=\sum_{j=0}^{n} a_{j} z^{j}$ be a polynomial of degree n. If $\operatorname{Re} a_{j}=\alpha_{j}$ and $\operatorname{Im} a_{j}=\beta_{j}$ for $j=0,1,2, \ldots, n, a_{n} \neq 0$ and if for some positive integer $\lambda \leq n$ and $k \geq 1$

$$
\begin{gathered}
k^{n-\lambda+1} \alpha_{n} \geq k^{n-\lambda} \alpha_{n-1} \geq k^{n-\lambda-1} \alpha_{n-2} \geq \ldots \geq k \alpha_{\lambda} \geq \alpha_{\lambda-1} \geq \ldots \geq \alpha_{1} \geq \alpha_{0} \\
\beta_{n} \geq \beta_{n-1} \geq \ldots \geq \beta_{1} \geq \beta_{0}>0
\end{gathered}
$$

then all the zeros of $\mathrm{P}(\mathrm{z})$ lie in

$$
\left|z+\frac{\alpha_{n}}{a_{n}}(k-1)\right| \leq \frac{\left\{\alpha_{n}-\alpha_{0}+\left|\alpha_{0}\right|+(k-1)\left(\sum_{i=\lambda}^{n}\left(\alpha_{j}+\left|\alpha_{j}\right|\right)-\left|\alpha_{n}\right|\right)+\beta_{n}\right\}}{\left|a_{n}\right|}
$$

Remark 3.1: If we take $\lambda=n$ in above we obtain Theorem D. 
Theorem 4: Let $P(z)=\sum_{j=0}^{n} a_{j} z^{j}$ be a polynomial of degree n. If for some positive integer $\lambda \leq n, k \geq 1$ and $\mu \geq 1$

$$
\begin{gathered}
k^{n-\lambda+1} \alpha_{n} \geq k^{n-\lambda} \alpha_{n-1} \geq k^{n-\lambda-1} \alpha_{n-2} \geq \ldots \geq k \alpha_{\lambda} \geq \alpha_{\lambda-1} \geq \ldots \geq \alpha_{1} \geq \alpha_{0} \\
\mu \beta_{n} \geq \beta_{n-1} \geq \ldots \geq \beta_{1} \geq \beta_{0}>0
\end{gathered}
$$

then all the zeros of $\mathrm{P}(\mathrm{z})$ lie in

$$
\left|z+\frac{k \alpha_{n}+i \mu \beta_{n}}{a_{n}}-1\right| \leq \frac{\left\{\alpha_{n}-\alpha_{0}+\left|\alpha_{0}\right|+(k-1)\left(\sum_{i=\lambda}^{n}\left(\alpha_{j}+\left|\alpha_{j}\right|\right)-\left|\alpha_{n}\right|\right)+\mu \beta_{n}\right\}}{\left|a_{n}\right|}
$$

If we take $\lambda=n$ in above we obtain the following:

Corollary 4.1: Let $P(z)=\sum_{j=0}^{n} a_{j} z^{j}$ be a polynomial of degree $n$. If for some positive integer $\lambda \leq n$ , $k \geq 1$ and $\mu \geq 1$

$$
\begin{gathered}
k \alpha_{n} \geq \alpha_{n-1} \geq \ldots \geq \alpha_{1} \geq \alpha_{0} \\
\mu \beta_{n} \geq \beta_{n-1} \geq \ldots \geq \beta_{1} \geq \beta_{0}>0
\end{gathered}
$$

then all the zeros of $P(z)$ lie in

$$
\left|z+\frac{k \alpha_{n}+i \mu \beta_{n}}{a_{n}}-1\right| \leq \frac{\left\{k \alpha_{n}-\alpha_{0}+\left|\alpha_{0}\right|+\mu \beta_{n}\right\}}{\left|a_{n}\right|}
$$

Theorem 5: Let $P(z)=\sum_{j=0}^{n} a_{j} z^{j}$ be a polynomial of degree n. If for some positive integer $\lambda \leq n$ and $k \geq 1$

$$
k^{n-\lambda+1}\left|a_{n}\right| \geq k^{n-\lambda}\left|a_{n-1}\right| \geq k^{n-\lambda-1}\left|a_{n-2}\right| \geq \ldots \geq k\left|a_{\lambda}\right| \geq\left|a_{\lambda-1}\right| \geq \ldots \geq\left|a_{1}\right| \geq\left|a_{0}\right|
$$

and for some real $\beta$,

$$
\left|\arg a_{j}-\beta\right| \leq \alpha \leq \frac{\pi}{2}, j=0,1,2, \ldots
$$

then all the zeros of lie in $|z+k-1| \leq k(\cos \alpha+\sin \alpha)+\frac{1}{\left|a_{n}\right|}\{(k-1) \cos \alpha+(k+1) \sin \alpha\} \sum_{i=\lambda+1}^{n}\left|a_{j}\right|+(k-1) \sum_{i=\lambda}^{n-1}\left|a_{i}\right|+\frac{2 \sin \alpha}{\left|a_{n}\right|} \sum_{j=\lambda-1}^{1}\left|a_{j}\right|$.

$$
\begin{aligned}
& F(z)=(1-z) P(z) \\
& =-a_{n} z^{n+1}+\left(a_{n}-a_{n-1}\right) z^{n}+\ldots+\left(a_{1}-a_{0}\right) z+a_{0} \\
& =\left\{-\alpha_{n} z^{n+1}+\left(\alpha_{n}-\alpha_{n-1}\right) z^{n}+\ldots+\left(\alpha_{1}-\alpha_{0}\right) z+\alpha_{0}\right\} \\
& +i\left\{-\beta_{n} z^{n+1}+\left(\beta_{n}-\beta_{n-1}\right) z^{n}+\ldots+\left(\beta_{1}-\beta_{0}\right) z+\beta_{0}\right\}
\end{aligned}
$$


A. Liman et al. / BIBECHANA 10 (2014) 71-81 : BMHSS, p.75 (Online Publication: Dec., 2013)

$$
\begin{aligned}
&=\{-\alpha_{n} z^{n}(z+k-1)+\left(k \alpha_{n}-\alpha_{n-1}\right) z^{n}+\left(k \alpha_{n-1}-\alpha_{n-2}\right) z^{n-1}+\ldots+\left(k \alpha_{\lambda+1}-\alpha_{\lambda}\right) z^{\lambda+1} \\
&+\left(k \alpha_{\lambda}-\alpha_{\lambda-1}\right) z^{\lambda}+\ldots+\left(\alpha_{1}-\alpha_{0}\right) z+\alpha_{0}-(k-1)\left(\alpha_{n-1} z^{n-1}+\alpha_{n-2} z^{n-2}\right. \\
&\left.\left.+\ldots+\alpha_{\lambda+1} z^{\lambda+1}+\alpha_{\lambda} z^{\lambda}\right)\right\}+i\left\{-\beta_{n} z^{n+1}+\left(\beta_{n}-\beta_{n-1}\right) z^{n}+\ldots+\left(\beta_{1}-\beta_{0}\right) z+\beta_{0}\right\} \\
&=-\alpha_{n} z^{n}(z+k-1)+\left(k \alpha_{n}-\alpha_{n-1}\right) z^{n}+\left(k \alpha_{n-1}-\alpha_{n-2}\right) z^{n-1}+\ldots+\left(k \alpha_{\lambda+1}-\alpha_{\lambda}\right) z^{\lambda+1} \\
&+\left(k \alpha_{\lambda}-\alpha_{\lambda-1}\right) z^{\lambda}+\ldots+\left(\alpha_{1}-\alpha_{0}\right) z+\alpha_{0}-(k-1)\left(\alpha_{n-1} z^{n-1}+\alpha_{n-2} z^{n-2}\right. \\
&\left.\left.\left.+\ldots+\alpha_{\lambda+1} z^{\lambda+1}+\alpha_{\lambda} z^{\lambda}\right)\right\}-i \beta_{n} z^{n+1}+i \beta_{0}+i \sum_{j=1}^{n}\left(\beta_{j}-\beta_{j-1}\right) z^{j}\right\} \\
&=z^{n}\left\{-\alpha_{n}(z+k-1)+\left(k \alpha_{n}-\alpha_{n-1}\right)+\left(k \alpha_{n-1}-\alpha_{n-2}\right) \frac{1}{z}+\ldots+\left(k \alpha_{\lambda+1}-\alpha_{\lambda}\right) \frac{1}{z^{n-\lambda-1}}+\left(k \alpha_{\lambda}-\alpha_{\lambda-1}\right) \frac{1}{z^{n-\lambda}}+\right. \\
&\left.+\ldots+\left(\alpha_{1}-\alpha_{0}\right) \frac{1}{z^{n-1}}+\alpha_{0} \frac{1}{z^{n}}-(k-1)\left\{\frac{\alpha_{n-1}}{z}+\frac{\alpha_{n-2}}{z^{2}}+\ldots+\frac{\alpha_{\lambda}}{z^{n-\lambda}}\right\}-i \beta_{n} z+i \frac{\beta_{0}}{z^{n}}+i \sum_{j=1}^{n}\left(\beta_{j}-\beta_{j-1}\right) \frac{1}{z^{n-j}}\right\}
\end{aligned}
$$

Let $|z|>1$ so that $\frac{1}{|z|^{n-j}}<1, j=0,1,2, \ldots$

$$
\begin{aligned}
F(z) \geq & |z|^{n}\left\{\left|\alpha_{n}\right||z+k-1|-\left[\left|k \alpha_{n}-\alpha_{n-1}\right|+\frac{\left|k \alpha_{n-1}-\alpha_{n-2}\right|}{|z|}+\ldots+\frac{\left|k \alpha_{\lambda+1}-\alpha_{\lambda}\right|}{|z|^{n-\lambda-1}}+\frac{\left|k \alpha_{\lambda}-\alpha_{\lambda-1}\right|}{|z|^{n-\lambda}+}\right.\right. \\
& \left.+\ldots+\frac{\left|\alpha_{1}-\alpha_{0}\right|}{|z|^{n-1}}+\frac{\left|\alpha_{0}\right|}{|z|^{n}}+|k-1| \mid \frac{\left|\alpha_{n-1}\right|}{|z|}+\frac{\left|\alpha_{n-2}\right|}{|z|^{2}}+\ldots+\frac{\left|\alpha_{\lambda+1}\right|}{|z|^{n-\lambda-1}}+\frac{\left|\alpha_{\lambda}\right|}{|z|^{n-\lambda}}\right) \\
& \left.\left.+\left|\beta_{n}\right||z|+\frac{\left|\beta_{0}\right|}{|z|^{n}}+\sum_{j=1}^{n}\left(\left|\beta_{j}\right|+\left|\beta_{j-1}\right|\right) \frac{1}{|z|^{n-j}}\right]\right\} \\
>|z|^{n}\left\{\left|\alpha_{n}\right||z+k-1|-\left[\left|k \alpha_{n}-\alpha_{n-1}\right|+\left|k \alpha_{n-1}-\alpha_{n-2}\right|+\ldots+\left|k \alpha_{\lambda+1}-\alpha_{\lambda}\right|+\left|k \alpha_{\lambda}-\alpha_{\lambda-1}\right|+\right.\right. & \\
& \left.\left.+\ldots+\left|\alpha_{1}-\alpha_{0}\right|+\left|\alpha_{0}\right|+|k-1| \sum_{j=\lambda}^{n-1}\left|\alpha_{j}\right|+\left|\beta_{n}\right|+\left|\beta_{0}\right|+\sum_{j=1}^{n}\left(\left|\beta_{j}\right|+\left|\beta_{j-1}\right|\right)\right]\right\} \\
=|z|^{n}\left\{\left|\alpha_{n}\right||z+k-1|-\left[\left|k \alpha_{n}-\alpha_{n-1}\right|+\left|k \alpha_{n-1}-\alpha_{n-2}\right|+\ldots+\left|k \alpha_{\lambda+1}-\alpha_{\lambda}\right|+\left|k \alpha_{\lambda}-\alpha_{\lambda-1}\right|+\right.\right. & \\
& \left.\left.+\ldots+\left|\alpha_{1}-\alpha_{0}\right|+\left|\alpha_{0}\right|+|k-1| \sum_{j=\lambda}^{n-1}\left|\alpha_{j}\right|+\left|\beta_{n}\right|+\left|\beta_{0}\right|+\sum_{j=1}^{n}\left(\left|\beta_{j}\right|+\left|\beta_{j-1}\right|\right)\right]\right\} \\
=|z|^{n}\left\{\left|\alpha_{n}\right||z+k-1|-\left[\left\{\alpha_{n}-\alpha_{0}+\left|\alpha_{0}\right|+(k-1)\left(\sum_{j=\lambda}^{n}\left(\alpha_{j}+\left|\alpha_{j}\right|\right)-\left|\alpha_{n}\right|\right)\right\}+2 \sum_{j=0}^{n}\left|\beta_{j}\right|\right]\right\} &
\end{aligned}
$$


$>0,{ }_{\text {if }}$

$$
|z+k-1|>\frac{\alpha_{n}-\alpha_{0}+\left|\alpha_{0}\right|+(k-1)\left(\sum_{j=\lambda}^{n}\left(\alpha_{j}+\left|\alpha_{j}\right|\right)-\left|\alpha_{n}\right|\right)+2 \sum_{j=0}^{n}\left|\beta_{j}\right|}{\left|\alpha_{n}\right|}
$$

This shows that those zeros of $F(z)$ for which $|z|>1$ lie in

$$
|z+k-1| \leq \frac{\alpha_{n}-\alpha_{0}+\left|\alpha_{0}\right|+(k-1)\left(\sum_{j=\lambda}^{n}\left(\alpha_{j}+\left|\alpha_{j}\right|\right)-\left|\alpha_{n}\right|\right)+2 \sum_{j=0}^{n}\left|\beta_{j}\right|}{\left|\alpha_{n}\right|}
$$

But those zeros of $F(z)$ for which $|z| \leq 1$ already satisfy the above equation. We conclude that all the zeros of $F(z)$ and hence of $P(z)$ lie in the disc

$$
|z+k-1| \leq \frac{\alpha_{n}-\alpha_{0}+\left|\alpha_{0}\right|+(k-1)\left(\sum_{i=\lambda}^{n}\left(\alpha_{j}+\left|\alpha_{j}\right|\right)-\left|\alpha_{n}\right|\right)+2 \sum_{j=0}^{n}\left|\beta_{j}\right|}{\left|\alpha_{n}\right|}
$$

This completes the proof.

Proof of Theorem 2: The proof follows on the same lines of Theorem 1.

Proof of Theorem 3: Consider a polynomial

$$
\begin{aligned}
F(z)= & (1-z) P(z) \\
= & -a_{n} z^{n+1}+\left(a_{n}-a_{n-1}\right) z^{n}+\ldots+\left(a_{1}-a_{0}\right) z+a_{0} \\
= & \left\{-a_{n} z^{n+1}+\left(\alpha_{n}-\alpha_{n-1}\right) z^{n}+\ldots+\left(\alpha_{1}-\alpha_{0}\right) z+\alpha_{0}\right\} \\
& \quad+i\left\{\left(\beta_{n}-\beta_{n-1}\right) z^{n}+\ldots+\left(\beta_{1}-\beta_{0}\right) z+\beta_{0}\right\} \\
=\{ & -a_{n} z^{n+1}-\alpha_{n} z^{n}(k-1)+\left(k \alpha_{n}-\alpha_{n-1}\right) z^{n}+\left(k \alpha_{n-1}-\alpha_{n-2}\right) z^{n-1}+\ldots+\left(k \alpha_{\lambda+1}-\alpha_{\lambda}\right) z^{\lambda+1} \\
& \quad+\left(k \alpha_{\lambda}-\alpha_{\lambda-1}\right) z^{\lambda}+\ldots+\left(\alpha_{1}-\alpha_{0}\right) z+\alpha_{0}-(k-1)\left(\alpha_{n-1} z^{n-1}+\alpha_{n-2} z^{n-2}\right. \\
& \left.\left.\quad+\ldots+\alpha_{\lambda+1} z^{\lambda+1}+\alpha_{\lambda} z^{\lambda}\right)\right\}+i\left\{\left(\beta_{n}-\beta_{n-1}\right) z^{n}+\ldots+\left(\beta_{1}-\beta_{0}\right) z+\beta_{0}\right\} \\
& \quad z^{n}\left\{-a_{n} z-\alpha_{n}(k-1)+\left(k \alpha_{n}-\alpha_{n-1}\right)+\left(k \alpha_{n-1}-\alpha_{n-2}\right) \frac{1}{z}+\ldots+\left(k \alpha_{\lambda+1}-\alpha_{\lambda}\right) \frac{1}{z^{n-\lambda-1}}\right. \\
& +\left(k \alpha_{\lambda}-\alpha_{\lambda-1}\right) \frac{1}{z^{n-\lambda}}+\ldots+\left(\alpha_{1}-\alpha_{0}\right) \frac{1}{z^{n-1}}+\alpha_{0} \frac{1}{z^{n}}-(k-1)\left\{\frac{\alpha_{n-1}}{z}+\frac{\alpha_{n-2}}{z^{2}}+\ldots+\frac{\alpha_{\lambda}}{z^{n-\lambda}}\right\}+ \\
& \left.+i\left\{\left(\beta_{n}-\beta_{n-1}\right)+\ldots+\left(\beta_{1}-\beta_{0}\right) \frac{1}{z^{n-1}}+\beta_{0} \frac{1}{z^{n}}\right\}\right\}
\end{aligned}
$$


A. Liman et al. / BIBECHANA 10 (2014) 71-81 : BMHSS, p.77 (Online Publication: Dec., 2013)

$$
\begin{aligned}
=z^{n}\{ & -a_{n} z-\alpha_{n}(k-1)+\left(k \alpha_{n}-\alpha_{n-1}\right)+\left(k \alpha_{n-1}-\alpha_{n-2}\right) \frac{1}{z}+\ldots+\left(k \alpha_{\lambda+1}-\alpha_{\lambda}\right) \frac{1}{z^{n-\lambda-1}} \\
& +\left(k \alpha_{\lambda}-\alpha_{\lambda-1}\right) \frac{1}{z^{n-\lambda}}+\ldots+\left(\alpha_{1}-\alpha_{0}\right) \frac{1}{z^{n-1}}+\alpha_{0} \frac{1}{z^{n}}-(k-1)\left\{\frac{\alpha_{n-1}}{z}+\frac{\alpha_{n-2}}{z^{2}}+\ldots+\frac{\alpha_{\lambda}}{z^{n-\lambda}}\right\} \\
& \left.+i\left\{\left(\beta_{n}-\beta_{n-1}\right)+\ldots+\left(\beta_{1}-\beta_{0}\right) \frac{1}{z^{n-1}}+\beta_{0} \frac{1}{z^{n}}\right\}\right\}
\end{aligned}
$$

Let $|z|>1$ so that $\frac{1}{|z|^{n-j}}<1, j=0,1,2, \ldots$

$$
\begin{aligned}
& |F(z)| \geq|z|^{n}\left\{\left|a_{n} z+(k-1) \alpha_{n}\right|-\left\{\left|k \alpha_{n}-\alpha_{n-1}\right|+\frac{\left|k \alpha_{n-1}-\alpha_{n-2}\right|}{|z|}+\ldots+\frac{\left|k \alpha_{\lambda}-\alpha_{\lambda-1}\right|}{|z|^{n-\lambda}}+\right.\right. \\
& +\frac{\left|\alpha_{1}-\alpha_{0}\right|}{|z|^{n-1}}+\frac{\left|\alpha_{0}\right|}{|z|^{n}}+(k-1)\left(\frac{\left|\alpha_{n-1}\right|}{|z|}+\frac{\left|\alpha_{n-2}\right|}{|z|^{2}}+\ldots+\frac{\left|\alpha_{n-2}\right|}{|z|^{2}}\right) \\
& \left.\left.+i\left(\left|\beta_{n}-\beta_{n-1}\right|+\ldots+\frac{\left|\beta_{1}-\beta_{0}\right|}{|z|^{n-1}}+\frac{\left|\beta_{0}\right|}{|z|^{n}}\right)\right\}\right\} \\
& =|z|^{n}\left[\left|a_{n}\right|\left|z+(k-1) \frac{\alpha_{n}}{a_{n}}\right|-\left\{\alpha_{n}-\alpha_{0}+\left|\alpha_{0}\right|+(k-1)\left(\sum_{i=\lambda}^{n}\left(\alpha_{j}+\left|\alpha_{j}\right|\right)-\left|\alpha_{n}\right|\right)+\beta_{n}\right\}\right]
\end{aligned}
$$

$>0$

if

$$
\left|z+(k-1) \frac{\alpha_{n}}{a_{n}}\right|>\frac{\left\{\alpha_{n}-\alpha_{0}+\left|\alpha_{0}\right|+(k-1)\left(\sum_{i=\lambda}^{n}\left(\alpha_{j}+\left|\alpha_{j}\right|\right)-\left|\alpha_{n}\right|\right)+\beta_{n}\right\}}{\left|a_{n}\right|}
$$

This shows that the zeros of $F(z)$, for which $|z|>1$ lie in

$$
\left|z+(k-1) \frac{\alpha_{n}}{a_{n}}\right| \leq \frac{\left\{\alpha_{n}-\alpha_{0}+\left|\alpha_{0}\right|+(k-1)\left(\sum_{i=\lambda}^{n}\left(\alpha_{j}+\left|\alpha_{j}\right|\right)-\left|\alpha_{n}\right|\right)+\beta_{n}\right\}}{\left|a_{n}\right|} .
$$

But those zeros of $F(z)$ for which $|z| \leq 1$ already satisfy the above equation. We conclude that all the zeros of $F(z)$ and hence of $P(z)$ lie in the disc

$$
\left|z+(k-1) \frac{\alpha_{n}}{a_{n}}\right| \leq \frac{\left\{\alpha_{n}-\alpha_{0}+\left|\alpha_{0}\right|+(k-1)\left(\sum_{i=\lambda}^{n}\left(\alpha_{j}+\left|\alpha_{j}\right|\right)-\left|\alpha_{n}\right|\right)+\beta_{n}\right\}}{\left|a_{n}\right|}
$$


Proof of Theorem 4: Consider a polynomial

$$
\begin{aligned}
& F(z)=(1-z) P(z) \\
& =(1-z)\left(a_{n} z^{n}+a_{n-1} z^{n-1}+\ldots+a_{1} z+a_{0}\right) \\
& =-a_{n} z^{n+1}+\left(a_{n}-a_{n-1}\right) z^{n}+\ldots+\left(a_{1}-a_{0}\right) z+a_{0} \\
& =\left\{-a_{n} z^{n+1}+\left(\alpha_{n}-\alpha_{n-1}\right) z^{n}+\ldots+\left(\alpha_{1}-\alpha_{0}\right) z+\alpha_{0}\right\} \\
& +i\left\{+\left(\beta_{n}-\beta_{n-1}\right) z^{n}+\ldots+\left(\beta_{1}-\beta_{0}\right) z+\beta_{0}\right\} \\
& =\left\{-a_{n} z^{n+1}-\alpha_{n} z^{n}(k-1)+\left(k \alpha_{n}-\alpha_{n-1}\right) z^{n}+\left(k \alpha_{n-1}-\alpha_{n-2}\right) z^{n-1}+\ldots+\left(k \alpha_{\lambda+1}-\alpha_{\lambda}\right) z^{\lambda+1}\right. \\
& +\left(k \alpha_{\lambda}-\alpha_{\lambda-1}\right) z^{\lambda}+\ldots+\left(\alpha_{1}-\alpha_{0}\right) z+\alpha_{0}-(k-1)\left(\alpha_{n-1} z^{n-1}+\alpha_{n-2} z^{n-2}+\ldots+\alpha_{\lambda+1} z^{\lambda+1}\right. \\
& \left.\left.+\alpha_{\lambda} z^{\lambda}\right)\right\}+i\left\{\left(\mu \beta_{n}+\beta_{n}-\mu \beta_{n}-\beta_{n-1}\right) z^{n}+\ldots+\left(\beta_{1}-\beta_{0}\right) z+\beta_{0}\right\} \\
& =z^{n}\left\{\left(-a_{n} z-\alpha_{n} k+\alpha_{n}-i \mu \beta_{n}+i \beta_{n}\right)+\left(k \alpha_{n}-\alpha_{n-1}\right)+\left(k \alpha_{n-1}-\alpha_{n-2}\right) \frac{1}{z}+\ldots\right. \\
& +\left(k \alpha_{\lambda}-\alpha_{\lambda-1}\right) \frac{1}{z^{n-\lambda}}++\left(\alpha_{1}-\alpha_{0}\right) \frac{1}{z^{n-1}}+\alpha_{0} \frac{1}{z^{n}}-(k-1)\left\{\frac{\alpha_{n-1}}{z}+\frac{\alpha_{n-2}}{z^{2}}+\ldots+\frac{\alpha_{\lambda}}{z^{n-\lambda}}\right\} \\
& \left.+i\left\{\left(\mu \beta_{n}-\beta_{n-1}\right)+\ldots+\left(\beta_{1}-\beta_{0}\right) \frac{1}{z^{n-1}}+\beta_{0} \frac{1}{z^{n}}\right\}\right\} \\
& =z^{n}\left[\left(-a_{n} z-\alpha_{n} k-i \mu \beta_{n}+a_{n}\right)+\left\{\left(\left(k \alpha_{n}-\alpha_{n-1}\right)+\left(k \alpha_{n-1}-\alpha_{n-2}\right) \frac{1}{z}+\ldots\right.\right.\right. \\
& \left.+\left(k \alpha_{\lambda}-\alpha_{\lambda-1}\right) \frac{1}{z^{n-\lambda}}+\ldots+\left(\alpha_{1}-\alpha_{0}\right) \frac{1}{z^{n-1}}+\alpha_{0} \frac{1}{z^{n}}\right)-(k-1)\left(\frac{\alpha_{n-1}}{z}+\frac{\alpha_{n-2}}{z^{2}}+\ldots \frac{\alpha_{\lambda}}{z^{n-\lambda}}\right) \\
& \left.+i\left(\left(\mu \beta_{n}-\beta_{n-1}\right)+\ldots+\left(\beta_{1}-\beta_{0}\right) \frac{1}{z^{n-1}}+\beta_{0} \frac{1}{z^{n}}\right)\right\}
\end{aligned}
$$

Let $|z|>1$ so that $\frac{1}{|z|^{n-j}}<1, j=0,1,2, \ldots$

$$
\begin{aligned}
|F(z)| \geq|z|^{n}\left[\left|a_{n}\right|\left|z+\frac{k \alpha_{n}+i \mu \beta_{n}}{a_{n}}-1\right|-\left\{\left|k \alpha_{n}-\alpha_{n-1}\right|+\left|k \alpha_{n-1}-\alpha_{n-2}\right|+\ldots+\left|k \alpha_{\lambda}-\alpha_{\lambda-1}\right|+\right.\right. \\
\quad \ldots+\left|\alpha_{1}-\alpha_{0}\right|+\left|\alpha_{0}\right|+(k-1)\left(\left|\alpha_{n-1}\right|+\left|\alpha_{n-2}\right|+\ldots+\left|\alpha_{\lambda}\right|\right) \\
\left.\left.+\left|\mu \beta_{n}-\beta_{n-1}\right|+\ldots+\left|\beta_{1}-\beta_{0}\right|+\left|\beta_{0}\right|\right\}\right] \\
=|z|^{n}\left[\left|a_{n}\right|\left|z+\frac{k \alpha_{n}+i \mu \beta_{n}}{a_{n}}-1\right|-\left\{\alpha_{n}-\alpha_{0}+\left|\alpha_{0}\right|+(k-1)\left(\sum_{i=\lambda}^{n}\left(\alpha_{j}+\left|\alpha_{j}\right|\right)-\left|\alpha_{n}\right|\right)+\mu \beta_{n}\right\}\right]
\end{aligned}
$$

$>0$, 


$$
\left|z+\frac{k \alpha_{n}+i \mu \beta_{n}}{a_{n}}-1\right|>\frac{\left\{\alpha_{n}-\alpha_{0}+\left|\alpha_{0}\right|+(k-1)\left(\sum_{i=\lambda}^{n}\left(\alpha_{j}+\left|\alpha_{j}\right|\right)-\left|\alpha_{n}\right|\right)+\mu \beta_{n}\right\}}{\left|a_{n}\right|}
$$

This shows that the zeros of $F(z)$, for which $|z|>1$ lie in

$$
\left|z+\frac{k \alpha_{n}+i \mu \beta_{n}}{a_{n}}-1\right| \leq \frac{\left\{\alpha_{n}-\alpha_{0}+\left|\alpha_{0}\right|+(k-1)\left(\sum_{i=\lambda}^{n}\left(\alpha_{j}+\left|\alpha_{j}\right|\right)-\left|\alpha_{n}\right|\right)+\mu \beta_{n}\right\}}{\left|a_{n}\right|} .
$$

But those zeros of $F(z)$ for which $|z| \leq 1$ already satisfy the above equation. We conclude that all the zeros of $F(z)$ and hence of $P(z)$ lie in the disc.

$$
\left|z+\frac{k \alpha_{n}+i \mu \beta_{n}}{a_{n}}-1\right| \leq \frac{\left\{\alpha_{n}-\alpha_{0}+\left|\alpha_{0}\right|+(k-1)\left(\sum_{i=\lambda}^{n}\left(\alpha_{j}+\left|\alpha_{j}\right|\right)-\left|\alpha_{n}\right|\right)+\mu \beta_{n}\right\}}{\left|a_{n}\right|}
$$

Proof of Theorem 5: Consider a polynomial

$$
\begin{aligned}
F(z)= & (1-z) P(z) \\
= & (1-z)\left(a_{n} z^{n}+a_{n-1} z^{n-1}+\ldots+a_{1} z+a_{0}\right) \\
= & -a_{n} z^{n+1}+\left(a_{n}-a_{n-1}\right) z^{n}+\ldots+\left(a_{1}-a_{0}\right) z+a_{0} \\
= & z^{n}\left\{-a_{n}(z+k-1)+\left(k a_{n}-a_{n-1}\right)+\left(k a_{n-1}-a_{n-2}\right) \frac{1}{z}+\ldots+\left(k a_{\lambda+1}-a_{\lambda}\right) \frac{1}{z^{n-\lambda-1}}\right. \\
& \left.\quad\left(k a_{\lambda}-a_{\lambda-1}\right) \frac{1}{z^{n-\lambda}}+\left(a_{1}-a_{0}\right) \frac{1}{z^{n-1}}+a_{0} \frac{1}{z^{n}}-(k-1)\left\{\frac{a_{n-1}}{z}+\frac{a_{n-2}}{z^{2}}+\ldots+\frac{a_{\lambda}}{z^{n-\lambda}}\right\}\right\}
\end{aligned}
$$

Let $|z|>1$ so that $\frac{1}{|z|^{n-j}}<1, j=0,1,2, \ldots$

$$
\begin{aligned}
|F(z)| \geq & |z|^{n}\left\{\left|a_{n}\right||z+k-1|-\left\{\left|k a_{n}-a_{n-1}\right|+\left|k a_{n-1}-a_{n-2}\right|+\ldots+\left|k a_{\lambda+1}-a_{\lambda}\right|\right.\right. \\
& \left.+\left|k a_{\lambda}-a_{\lambda-1}\right|+\ldots+\left|a_{1}-a_{0}\right|+\left|a_{0}\right|+(k-1)\left(\left|a_{n-1}\right|+\left|a_{n-2}\right|+\ldots+\left|a_{\lambda}\right|\right)\right\} .
\end{aligned}
$$

Using the fact (for reference see [2]) that for any two complex numbers $b_{0}$ and $b_{1}$ such that $\left|b_{0}\right| \geq\left|b_{1}\right|$, and $\left|\arg a_{j}-\beta\right| \leq \alpha \leq \frac{\pi}{2}, j=0,1,2$ and $\beta$ real

$$
\left|b_{0}-b_{1}\right| \leq\left(\left|b_{0}\right|-\left|b_{1}\right|\right) \cos \alpha+\left(\left|b_{0}\right|+\left|b_{1}\right|\right) \sin \alpha \text {. }
$$




$$
\begin{aligned}
& |F(z)| \geq|z|^{n}\left[\left|a_{n}\right||z+k-1|-\left\{k\left|a_{n}\right| \cos \alpha-\left|a_{n-1}\right| \cos \alpha+k\left|a_{n}\right| \sin \alpha+\left|a_{n-1}\right| \sin \alpha\right.\right. \\
& +k\left|a_{n-1}\right| \cos \alpha-\left|a_{n-2}\right| \cos \alpha+k\left|a_{n-1}\right| \sin \alpha+\left|a_{n-2}\right| \sin \alpha+\ldots+k\left|a_{\lambda}\right| \cos \alpha \\
& -\left|a_{\lambda-1}\right| \cos \alpha+k\left|a_{\lambda}\right| \sin \alpha+\left|a_{\lambda-1}\right| \sin \alpha+\ldots+\left|a_{\lambda-1}\right| \cos \alpha-\left|a_{\lambda-2}\right| \cos \alpha+\left|a_{\lambda-1}\right| \sin \alpha \\
& \left.\left.+\left|a_{\lambda-2}\right| \sin \alpha+\ldots+\left|a_{1}\right| \cos \alpha-\left|a_{0}\right| \cos \alpha+\left|a_{1}\right| \sin \alpha+\left|a_{0}\right| \sin \alpha+\ldots+\left|a_{0}\right|+(k-1) \sum_{i=\lambda}^{n-1}\left|a_{i}\right|\right\}\right] \\
& =|z|^{n}\left[\left|a_{n}\right||z+k-1|-\left\{k\left|a_{n}\right|(\cos \alpha+\sin \alpha)+(k-1)\left|a_{n-1}\right| \cos \alpha+(k+1)\left|a_{n-1}\right| \sin \alpha\right.\right. \\
& +\ldots+(k-1)\left|a_{\lambda}\right| \cos \alpha+(k+1)\left|a_{\lambda}\right| \sin \alpha+2\left|a_{\lambda-1}\right| \sin \alpha+2\left|a_{\lambda-2}\right| \sin \alpha+\ldots \\
& \left.\left.+2\left|a_{1}\right| \sin \alpha-\left|a_{0}\right| \cos \alpha+\left|a_{0}\right| \sin \alpha+\left|a_{0}\right|+(k-1) \sum_{i=\lambda}^{n-1}\left|a_{i}\right|\right\}\right] \\
& =|z|^{n}\left[\left|a_{n}\right||z+k-1|-\left\{k\left|a_{n}\right|(\cos \alpha+\sin \alpha)+(k-1)\left(\cos \alpha \sum_{i=\lambda+1}^{n}\left|a_{j-1}\right|+\sum_{i=\lambda}^{n-1}\left|a_{i}\right|\right)\right.\right. \\
& \left.\left.+(k+1) \sin \alpha \sum_{i=\lambda+1}^{n}\left|a_{j-1}\right|+2 \sin \alpha \sum_{j=\lambda-1}^{1}\left|a_{j}\right|+\left|a_{0}\right|(\sin \alpha-\cos \alpha)+\left|a_{0}\right|\right\}\right] \\
& =|z|^{n}\left[\left|a_{n}\right||z+k-1|-\left\{k\left|a_{n}\right|(\cos \alpha+\sin \alpha)+((k-1) \cos \alpha+(k+1) \sin \alpha) \sum_{i=\lambda+1}^{n}\left|a_{j}\right|\right.\right. \\
& \left.\left.+2 \sin \alpha \sum_{j=\lambda-1}^{1}\left|a_{j}\right|+\left|a_{0}\right|(1+\sin \alpha-\cos \alpha)+(k-1) \sum_{i=\lambda}^{n-1}\left|a_{i}\right|\right\}\right] \\
& \geq|z|^{n}\left\{\left|a_{n}\right||z+k-1|-\left\{k\left|a_{n}\right|(\cos \alpha+\sin \alpha)+((k-1) \cos \alpha+(k+1) \sin \alpha) \sum_{i=\lambda+1}^{n}\left|a_{j}\right|\right.\right. \\
& \left.\left.+2 \sin \alpha \sum_{j=\lambda-1}^{1}\left|a_{j}\right|+(k-1) \sum_{i=\lambda}^{n-1}\left|a_{i}\right|\right\}\right\}
\end{aligned}
$$

$>0$,

if

$|z+k-1|>k(\cos \alpha+\sin \alpha)+\frac{1}{\left|a_{n}\right|}\{(k-1) \cos \alpha+(k+1) \sin \alpha\} \sum_{i=\lambda+1}^{n}\left|a_{j}\right|+\frac{2 \sin \alpha}{\left|a_{n}\right|} \sum_{j=\lambda-1}^{1}\left|a_{j}\right|+(k-1) \sum_{i=\lambda}^{n-1}\left|a_{i}\right|$

This shows that the zeros of $F(z)$, for which $|z|>1$ lie in 
A. Liman et al. / BIBECHANA 10 (2014) 71-81 : BMHSS, p.81 (Online Publication: Dec., 2013)

$|z+k-1| \leq k(\cos \alpha+\sin \alpha)+\frac{1}{\left|a_{n}\right|}\{(k-1) \cos \alpha+(k+1) \sin \alpha\} \sum_{i=\lambda+1}^{n}\left|a_{j}\right|+\frac{2 \sin \alpha}{\left|a_{n}\right|} \sum_{j=\lambda-1}^{1}\left|a_{j}\right|+(k-1) \sum_{i=\lambda}^{n-1}\left|a_{i}\right|$.

But those zeros of $F(z)$ for which $|z| \leq 1$ already satisfy the above equation the above equation. We conclude that all the zeros of $F(z)$ and hence of $P(z)$ lie in the disc

$|z+k-1| \leq k(\cos \alpha+\sin \alpha)+\frac{1}{\left|a_{n}\right|}\{(k-1) \cos \alpha+(k+1) \sin \alpha\} \sum_{i=\lambda+1}^{n}\left|a_{j}\right|+\frac{2 \sin \alpha}{\left|a_{n}\right|} \sum_{j=\lambda-1}^{1}\left|a_{j}\right|+(k-1) \sum_{i=\lambda}^{n-1}\left|a_{i}\right|$.

\section{References}

1. M. Marden, Geometry of Polynomials, Math. Surveys, No. 3; Amer. Math. Soc. Providence R.I., (1966).

2. Q. I. Rahman and G. Schmeisser, Analytic Theory of Polynomials. Oxford Univ. Press, (2002).

3. W.M Shah and A. Liman, On Eneström Kakeya Theorem and related Analytic Functions, Proc. Indian Acad. Sci.( Math Sci.) vol.117,No. 3,(2007) 359-370.

4. A. Joyal, G. Labelle and Q.I-Rahman, On the location of zeros of polynomials, Canad. Math. Bull.,10(1967) 53-63.

5. A.Aziz and B.A Zargar, Some extension of Eneström-Kakeya theorem,Glasnik Matematicki, 31(1996) 239-244. 